PROCEEDINGS OF THE

AMERICAN MATHEMATICAL SOCIETY

Volume 129, Number 7 , Pages 2039-2044

S 0002-9939(00)05771-3

Article electronically published on November 30, 2000

\title{
COMPOSITION OPERATORS WITH CLOSED RANGE ON THE BLOCH SPACE
}

\author{
PRATIBHA GHATAGE, JUN YAN, AND DECHAO ZHENG
}

(Communicated by Joseph A. Ball)

\begin{abstract}
In this note we investigate conditions under which a holomorphic self-map of the unit disk induces a composition operator with closed range on the Bloch space.
\end{abstract}

\section{InTRODUCTION}

For each $z \in D$, let $\varphi_{z}$ denote the Möbius transformation of $D$

$$
\varphi_{z}(w)=\frac{z-w}{1-\bar{z} w},
$$

for $w \in D$. The pseudo-hyperbolic distance on $D$ is defined by

$$
\rho(z, w)=\left|\varphi_{z}(w)\right|, \quad z, w \in D .
$$

The pseudohyperbolic distance is Möbius invariant, that is,

$$
\rho(g z, g w)=\rho(z, w),
$$

for all $g \in \operatorname{Aut}(D)$, the Möbius group of $D$, and all $z, w \in D$. It has the following useful property:

$$
1-\rho(z, w)^{2}=\frac{\left(1-|z|^{2}\right)\left(1-|w|^{2}\right)}{|1-\bar{z} w|^{2}} .
$$

A function $f$ is called a Bloch function if it is analytic in $D$ and if

$$
\|f\|_{B}=\sup _{z \in D}\left(1-|z|^{2}\right)\left|f^{\prime}(z)\right|<\infty .
$$

This defines a seminorm, and the Bloch functions form a complex Banach space $B$ with the norm

$$
\|f\|_{\beta}=|f(0)|+\|f\|_{B} .
$$

We will show that $\left(1-|z|^{2}\right)\left|f^{\prime}(z)\right|$ is Lipschitz with respect to pseudo-hyperbolic metric and use this result to study composition operators on the Bloch space in Section 3

The little Bloch space of $D$, denoted $B_{0}$, is the closed subspace of $B$ consisting of functions $f$ with $\left(1-|z|^{2}\right)\left|f^{\prime}(z)\right| \rightarrow 0$ as $|z| \rightarrow 1^{-}$.

Received by the editors May 25, 1999 and, in revised form, November 12, 1999.

2000 Mathematics Subject Classification. Primary 46E15.

Supported in part by the National Science Foundation and University Research Council of Vanderbilt University. 
The composition operator $C_{\varphi}: H(D) \rightarrow H(D)$ is defined by $C_{\varphi} f=f \circ \varphi$ on the space of analytic funtions on $D$. It is well known exactly when $C_{\varphi}$ is compact on $B$ or $B_{0}$ [2]. To our knowledge no such study has been done to determine when $C_{\varphi}$ has closed range. In this note we give a set of necessary conditions and a partial converse. For a comprehensive treatment of composition operators we refer the reader to 3 .

We are grateful to Joel Shapiro for helpful discussions and to Wayne Smith for pointing out an error in our work and bringing the relavent part of his work to our notice. The third author also thanks Chongqing University and Sichuan University, for their hospitality while part of this work was in progress.

\section{NECESSARY CONDITIONS}

We assume that $\varphi$ is a holomorphic self-map of the unit disk $D$ and $C_{\varphi}$ is the composition operator on the Bloch space $B$. We write $G=\varphi(D)$, and $\tau_{\varphi}(z)=$ $\left(1-|z|^{2}\right) \varphi^{\prime}(z) /\left(1-|\varphi(z)|^{2}\right)$. The following are two simple consequences of the Schwarz-Pick Lemma [1, p. 2].

(1) $C_{\varphi}$ maps $B$ into $B$.

(2) $0 \leq\left|\tau_{\varphi}(z)\right| \leq 1$.

Lemma 1. If $C_{\varphi}$ is bounded below on $B$, then, for all $f \in B$,

$$
\|f\|_{B} \leq k\left\{\sup \left(1-|w|^{2}\right)\left|f^{\prime}(w)\right|, w \in G\right\}
$$

for some $k$.

The proof is a trivial consequence of (2) above.

As Smith has shown in 4] there exists a univalent function $\varphi: D \rightarrow D$ with $\varphi(D)$ dense in $D$ for which $\tau_{\varphi}(z) \rightarrow 0$ as $|z| \rightarrow 1$ [4, 6.5]. This is known to be equivalent to the fact that $C_{\varphi}$ is compact on $B_{0}[2]$.

Proposition 1. If $C_{\varphi}$ is bounded below on $B$, then there exist positive constants $\epsilon, r$ with $r<1$ such that, for all $z \in D, \rho\left(\varphi\left(\Omega_{\epsilon}\right), z\right) \leq r$ where $\Omega_{\epsilon}=\left\{z \in D,\left|\tau_{g}(z)\right|>\epsilon\right\}$.

Proof. Since $C_{\varphi}: B \rightarrow B$ is bounded below, there is a constant $0<k \leq 1$ such that

$$
\left\|C_{\varphi} f\right\|_{\beta} \geq k\|f\|_{\beta},
$$

for $f \in B_{0}$. For each $w \in D$, let $f_{w}(z)=\frac{w-z}{1-\bar{w} z}-\frac{w-\varphi(0)}{1-\bar{w} \varphi(0)}$. Clearly, $f_{w}$ is a bounded and continuous analytic function on the closed unit disk and so is in $B_{0}$. Moreover, an easy computation gives $\left\|f_{w}\right\|_{\beta} \geq 1$. Thus

$$
\left\|C_{\varphi} f_{w}\right\|_{\beta} \geq k\left\|f_{w}\right\|_{\beta}=k .
$$

On the other hand, we also have

$$
\left(1-|z|^{2}\right)\left|\left(C_{\varphi} f_{w}\right)^{\prime}(z)\right|=\left(1-\left|\varphi_{w}(\varphi(z))\right|^{2}\right)\left|\tau_{\varphi}(z)\right|,
$$

and $C_{\varphi} f_{w}(0)=0$. Then there is a point $z_{w} \in D$ such that

$$
\left(1-\left|z_{w}\right|^{2}\right)\left(C_{\varphi} f_{w}\right)^{\prime}\left(z_{w}\right) \mid \geq\left\|C_{\varphi} f_{w}\right\|_{\beta} / 2 \geq k / 2 .
$$

So we obtain that

$$
\left(1-\left|\varphi_{w}\left(\varphi\left(z_{w}\right)\right)\right|^{2}\right)\left|\tau_{\varphi}\left(z_{w}\right)\right| \geq k / 2
$$


Thus

$$
\left(1-\left|\varphi_{w}\left(\varphi\left(z_{w}\right)\right)\right|^{2}\right) \geq k / 2,
$$

and

$$
\left|\tau_{\varphi}\left(z_{w}\right)\right| \geq k / 2
$$

so

$$
\left|\varphi_{w}\left(\varphi\left(z_{w}\right)\right)\right|^{2} \leq 1-k / 2
$$

Let $r=\sqrt{1-k / 2}<1$, and $\epsilon=k / 2$. Noting $\rho\left(\varphi\left(z_{w}\right), w\right)=\left|\varphi_{w}\left(\varphi\left(z_{w}\right)\right)\right|$, we conclude that

$$
\begin{gathered}
\rho\left(\varphi\left(z_{w}\right), w\right)<r, \text { and } \\
\left|\tau_{\varphi}\left(z_{w}\right)\right| \geq \epsilon .
\end{gathered}
$$

This completes the proof.

\section{Sufficient CONDitions}

In this section we will obtain a sufficient condition for $C_{\varphi}$ to be bounded below on the Bloch space. First we show that for a Bloch function $f,\left(1-|z|^{2}\right)\left|f^{\prime}(z)\right|$ is Lipschitz.

Theorem 1. Let $f$ be in the Bloch space. Then

$$
\left|\left(1-|z|^{2}\right)\right| f^{\prime}(z)\left|-\left(1-|w|^{2}\right)\right| f^{\prime}(w)|| \leq 3.31 \rho(z, w)\|f\|_{B},
$$

for $z, w \in D$.

Proof. For $z, w$ in $D$, let $\lambda=\varphi_{w}(z)$. Then

$$
\left(1-|w|^{2}\right) f^{\prime}(w)=\left(f \circ \varphi_{w}\right)^{\prime}(0)
$$

and

$$
\left(1-|z|^{2}\right)\left|\left(f \circ \varphi_{w}\right)^{\prime}(z)\right|=\left(1-|\lambda|^{2}\right)\left|f^{\prime}(\lambda)\right| .
$$

Letting $g=f \circ \varphi_{w}$, we can rewrite the above equations as

$$
g^{\prime}(0)=\left(1-|w|^{2}\right) f^{\prime}(w),
$$

and

$$
\left(1-|\lambda|^{2}\right)\left|g^{\prime}(\lambda)\right|=\left(1-|z|^{2}\right)\left|\left(f \circ \varphi_{w}\right)^{\prime}(z)\right| .
$$

Thus

$$
\begin{gathered}
\left|\left(1-|z|^{2}\right)\right| f^{\prime}(z)\left|-\left(1-|w|^{2}\right)\right| f^{\prime}(w)|| \\
=\left|\left(1-|\lambda|^{2}\right)\right| g^{\prime}(\lambda)|-| g^{\prime}(0)|| \\
=|\lambda|^{2}\left|g^{\prime}(0)\right|+\left(1-|\lambda|^{2}\right)\left|g^{\prime}(\lambda)-g^{\prime}(0)\right| .
\end{gathered}
$$

Clearly by the definition of $\|g\|_{B}$ we have

$$
\left|g^{\prime}(0)\right| \leq\|g\|_{B}=\left\|f \circ \varphi_{w}\right\|_{B}=\|f\|_{B},
$$

where the last equation comes from the fact that the Bloch norm is the Möbius invariant. Now we turn to estimate $\left|g^{\prime}(\lambda)-g^{\prime}(0)\right|$. To do this, let $\lambda \in D$. 
Note, for each $z \in D$,

$$
\begin{aligned}
& \left(1-|z|^{2}\right) g^{\prime \prime}(z)=\left(g^{\prime} \circ \varphi_{z}\right)^{\prime}(0) \\
= & \frac{1}{2 \pi r} \int_{0}^{2 \pi} g^{\prime} \circ \varphi_{z}\left(r e^{i \theta}\right) e^{-i \theta} d \theta,
\end{aligned}
$$

for all $0<r<1$. Then for each fixed $0<r<1$, we have

$$
\begin{gathered}
\left|\int_{0}^{2 \pi} g^{\prime} \circ \varphi_{z}\left(r e^{i \theta}\right) e^{-i \theta} d \theta\right| \\
\leq \int_{0}^{2 \pi} \frac{1-\left|\varphi_{z}\left(r e^{i \theta}\right)\right|^{2}}{1-\left|\varphi_{z}\left(r e^{i \theta}\right)\right|^{2}}\left|g^{\prime} \circ \varphi_{z}\left(r e^{i \theta}\right)\right| d \theta \\
\leq\|g\|_{B} \int_{0}^{2 \pi} \frac{1}{1-\left|\varphi_{z}\left(r e^{i \theta}\right)\right|^{2}} d \theta .
\end{gathered}
$$

On the other hand, we also have

$$
\frac{1}{1-\left|\varphi_{z}\left(r e^{i \theta}\right)\right|^{2}}=\frac{\left|1-r e^{i \theta} \bar{z}\right|^{2}}{\left(1-|z|^{2}\right)\left(1-r^{2}\right)}
$$

Thus we obtain

$$
\left(1-|z|^{2}\right)^{2}\left|g^{\prime \prime}(z)\right| \leq\|g\|_{B} \frac{1+r^{2}|z|^{2}}{r\left(1-r^{2}\right)}
$$

for $0<r<1$.

Now we consider two cases.

Case 1. If $|\lambda| \leq 1 / 2$, letting $h(r)=\frac{1+r^{2} / 4}{r\left(1-r^{2}\right)}$, then the minimal value of $h(r)$ for $0<r<1$ approximately equals 2.81 . Hence for $|z| \leq 1 / 2$, we have

$$
\left(1-|z|^{2}\right)^{2}\left|g^{\prime \prime}(z)\right| \leq 2.81\|g\|_{B}
$$

Also, $\left|g^{\prime}(\lambda)-g^{\prime}(0)\right| \leq \int_{0}^{1}\left|g^{\prime \prime}(t \lambda)\right||\lambda| d t$. Hence

$$
\left|g^{\prime}(\lambda)-g^{\prime}(0)\right| \leq 2.81\|g\|_{B} \int_{0}^{1} \frac{|\lambda| d t}{\left(1-t^{2}|\lambda|^{2}\right)^{2}}=\int_{0}^{|\lambda|} \frac{d s}{\left(1-s^{2}\right)^{2}}
$$

An easy calculation gives

$$
\int_{0}^{|\lambda|} \frac{d s}{\left(1-s^{2}\right)^{2}}=\frac{1}{4}\left[\frac{2|\lambda|}{1-|\lambda|^{2}}+\ln \frac{1+|\lambda|}{1-|\lambda|}\right]
$$

So we get

$$
\left(1-|\lambda|^{2}\right)\left|g^{\prime}(\lambda)-g^{\prime}(0)\right| \leq 2.81\|g\|_{B}|\lambda|=2.81\|f\|_{B}|\lambda| .
$$

Thus if $|\lambda| \leq 1 / 2$, we have

$$
\left|\left(1-|\lambda|^{2}\right)\right| g^{\prime}(\lambda)|-| g^{\prime}(0)\left\|\leq|\lambda|^{2}\right\| f\left\|_{B}+2.81|\lambda|\right\| f\left\|_{B} \leq 3.31|\lambda|\right\| f \|_{B} .
$$


Case 2. If $|\lambda|>1 / 2$, then $2|\lambda|>1$. In this case,

$$
\begin{gathered}
\left|\left(1-|\lambda|^{2}\right)\right| g^{\prime}(\lambda)|-| g^{\prime}(0) \| \\
\leq \max \left\{\left(1-|\lambda|^{2}\right)\left|g^{\prime}(\lambda)\right|,\left|g^{\prime}(0)\right|\right\} \leq\|g\|_{B}=\|f\|_{B} \leq 2|\lambda|\|f\|_{B} .
\end{gathered}
$$

Combining the above two cases we have

$$
\left|\left(1-|\lambda|^{2}\right)\right| g^{\prime}(\lambda)|-| g^{\prime}(0)\|\leq 3.31|\lambda|\| f \|_{B},
$$

for $|\lambda|<1$. Hence we conclude that

$$
\begin{gathered}
\left|\left(1-|z|^{2}\right)\right| f^{\prime}(z)\left|-\left(1-|w|^{2}\right)\right| f^{\prime}(w)||=\left|\left(1-|\lambda|^{2}\right)\right| g^{\prime}(\lambda)|-| g^{\prime}(0)|| \\
\leq 3.31|\lambda|\|f\|_{B}=3.31 \mid \rho(z, w)\|f\|_{B},
\end{gathered}
$$

noting that $\rho(z, w)=|\lambda|$. This completes the proof.

Theorem 2. If for some constants $0<r<1 / 4$, and $\epsilon>0$, for each $w \in D$, there is a point $z_{w} \in D$ such that

$$
\rho\left(\varphi\left(z_{w}\right), w\right)<r \text { and }\left|\tau_{\varphi}\left(z_{w}\right)\right|>\epsilon,
$$

then $C_{\varphi}: B \rightarrow B$ is bounded below.

Proof. Let $\lambda=\varphi(0)$. Then $\varphi=\varphi_{\lambda} \circ \varphi_{\lambda} \circ \varphi$. Let $\psi=\varphi_{\lambda} \circ \varphi$. Thus $\psi(0)=0$, and $C_{\varphi}=C_{\psi} C_{\varphi_{\lambda}}$. Since $\varphi_{\lambda}$ is a Möbius transform, $C_{\varphi_{\lambda}}$ is an isometry on $B$. So we need only to prove that $C_{\psi}$ is bounded below on the Bloch space. Moreover $\psi$ still satisfies the conditions of the theorem.

In order to prove that $C_{\psi}$ is bounded below on the Bloch space it suffices to prove

$$
\left\|C_{\psi} f\right\|_{\beta} \geq k
$$

for some constant $k>0$ and all $f \in B$ with $\|f\|_{\beta}=1$. To do this, let $f$ be a function in the Bloch space with norm 1. For each $z \in D$, we have

$$
\begin{gathered}
\left(1-|z|^{2}\right)\left|\left(C_{\psi} f\right)^{\prime}(z)\right| \\
=\left(1-|\psi(z)|^{2}\right)\left|f^{\prime}(\psi(z))\right| \tau_{\psi}(z) \mid .
\end{gathered}
$$

Since $\|f\|_{\beta}=1$, there is a point $w \in D$ such that

$$
\left(1-|w|^{2}\right)\left|f^{\prime}(w)\right| \geq(1-(1 / 4-r) / 2)(1-|f(0)|) .
$$

By Theorem 1, we have

$$
\left|\left(1-|w|^{2}\right)\right| f^{\prime}(w)\left|-\left(1-|z|^{2}\right)\right| f^{\prime}(z)|| \leq 4 \rho(z, w)(1-|f(0)|) .
$$

Thus whenever $\rho\left(\psi\left(z_{w}\right), w\right)<r<1 / 4$, we have that

$$
\begin{aligned}
& \left(1-\left|\psi\left(z_{w}\right)\right|^{2}\right)\left|f^{\prime}\left(\psi\left(z_{w}\right)\right)\right| \geq\left(1-|w|^{2}\right)\left|f^{\prime}(w)\right|-4 r(1-|f(0)|) \\
& \geq[(1-(1 / 4-r) / 2)-4 r](1-|f(0)|)=[7(1-4 r) / 8](1-|f(0)|) .
\end{aligned}
$$

So

$$
\begin{gathered}
\left\|C_{\psi} f\right\|_{\beta} \geq|f(\psi(0))|+\left(1-|z|^{2}\right)\left|\left(C_{\psi} f\right)^{\prime}(z)\right| \\
\geq|f(0)|+\left(1-|\psi(z)|^{2}\right)\left|f^{\prime}(\psi(z))\right|\left|\tau_{\psi}(z)\right|,
\end{gathered}
$$


for all $z \in D$. In particular,

$$
\begin{aligned}
& \left\|C_{\psi} f\right\|_{\beta} \geq|f(0)|+\left(1-\left|\psi\left(z_{w}\right)\right|^{2}\right)\left|f^{\prime}\left(\psi\left(z_{w}\right)\right)\right|\left|\tau_{\psi}\left(z_{w}\right)\right| \\
& \geq|f(0)|+[7(1-4 r) \epsilon / 8](1-|f(0)|) \geq[7(1-4 r) \epsilon / 8] .
\end{aligned}
$$

Let $k=[7(1-4 r) \epsilon / 8]$. We have proved that

$$
\left\|C_{\psi} f\right\|_{\beta} \geq k
$$

whenever $\|f\|_{\beta}=1$. This completes the proof.

\section{ADDENDUM}

As an expected corollary to Theorem 1 we get a converse to the RochbergSemmes result on interpolation by derivatives of Bloch functions. An easy exposition of it is found in Proposition 1 of [2] along with more references to similar work.

Corollary 1. If a sequence $\left\{z_{n}\right\} \subseteq D$ satisfies the property that the map $S: B \rightarrow$ $\ell^{\infty}$ defined by $S(f)=\left\{\left(1-\left|z_{n}\right|^{2}\right) f^{\prime}\left(z_{n}\right)\right\}$ is onto, then $\left\{z_{n}\right\}$ is separated in the pseudo-hyperbolic metric.

Proof. For a fixed $n$ choose $f_{n} \epsilon B$ with $\left\|f_{n}\right\|_{B} \leq c,\left(1-\left|z_{n}\right|^{2}\right) f_{n}^{\prime}\left(z_{n}\right)=1$ and $\left(1-\left|z_{m}\right|^{2}\right) f_{n}^{\prime}\left(z_{m}\right)=0$ whenever $m \neq n$. Then by Theorem $1,1 \leq 4 c \rho\left(z_{n}, z_{m}\right) \forall n \neq$ $m$.

We are grateful to Richard Rochberg for pointing out the connection.

\section{FINAL REMARK}

The preliminary investigations in this paper have led to stronger results including a set of necessary and sufficient conditions and their geometric interpretation. They will appear elsewhere.

\section{REFERENCES}

[1] J. Garnett, Bounded Analytic Functions, Academic Press, New York, 1981. MR 83g:30037

[2] K. Madigan and A. Matheson, Compact Composition operators on the Bloch space, Trans. Amer. Math. Soc. 347(1995). 2679-2687. MR 95i:47061

[3] J. Shapiro, Composition Operators and Classical Function Theory. Spring-Verlag, New York, 1993. MR 94k:47049

[4] W. Smith, Composition operators between Bergman and Hardy spaces, Trans. Amer. Math. Soc. 348(1996), 2331-2348. MR 96i:47056

Department of Mathematics, Cleveland State University, Cleveland, Ohio 44115

E-mail address: ghatage@math.csuohio.edu

Department of Mathematics, Chongqing University, Chongqing, People's Republic OF CHINA

Department of Mathematics, Vanderbilt University, Nashville, Tennessee 37240

E-mail address: zheng@math.vanderbilt.edu 